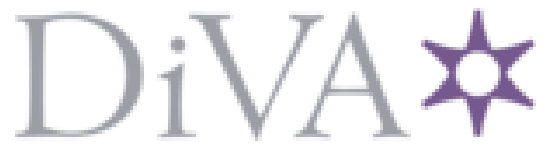

http://www.diva-portal.org

Postprint

This is the accepted version of a paper presented at 2017 IEEE 20th International Conference on Intelligent Transportation Systems (ITSC).

Citation for the original published paper:

Henriksson, M., Flärdh, O., Mårtensson, J. (2017)

Optimal Powertrain Control of a Heavy-Duty Vehicle Under Varying Speed Requirements.

In: IEEE

N.B. When citing this work, cite the original published paper.

(C)2017 IEEE

Permanent link to this version:

http://urn.kb.se/resolve?urn=urn:nbn:se:kth:diva-220134 


\section{Optimal Powertrain Control of a Heavy-Duty Vehicle Under Varying Speed Requirements}

\author{
Manne Henriksson \\ Department of Automatic Control \\ KTH Royal Institute of Technology \\ and Scania CV AB \\ Email: manneh@kth.se
}

\author{
Oscar Flärdh \\ Scania CV AB \\ 15187 Södertälje, Sweden \\ Email: oscar.flardh@scania.com
}

\author{
Jonas Mårtensson \\ Department of Automatic Control \\ and the Integrated Transport Research Lab \\ KTH Royal Institute of Technology \\ 10044 Stockholm, Sweden. Email: jonas1@kth.se
}

\begin{abstract}
Reducing the fuel consumption is a major issue in the vehicle industry. In this paper, it is done by formulating a driving mission of a heavy-duty truck as an optimal control problem and solving it using dynamic programming. The vehicle model includes an engine and a gearbox with parameters based on measurements in test cells. The dynamic programming algorithm is solved by considering four specific types of transitions: transitions between the same gear, coasting in neutral gear, coasting with a gear engaged with no fuel injection and transitions involving gear changes. Simulations are performed on a driving cycle commonly used for testing distribution type of driving. In order to make sure that the truck does not deviate too much from a normal way of driving, restrictions on maximum and minimum allowed velocities are imposed based on statistics from real traffic data. The simulations show that $12.7 \%$ fuel can be saved without increasing the trip time by allowing the truck to engage neutral gear and make small deviations from the reference trajectory.
\end{abstract}

\section{INTRODUCTION}

Manufacturers of heavy-duty trucks are constantly striving to reduce the fuel consumption of their vehicles. One way of doing so is by improving the hardware of the vehicle, e.g. by increasing the efficiency of the engine or the gearbox. Another way, which is the focus of this paper, is by improving the software. An example of such a solution is the lookahead cruise controller, which takes future road topography into consideration when controlling the velocity. An average fuel saving of $3 \%$ has been reported [1]. This kind of solution today mainly considers highway driving where the desired cruising velocity is constant.

Fuel efficient ways of driving is often found by formulating the driving mission as an optimal control problem, see [2] for an overview of such methods in automotive applications. In [3], the optimal control of a heavy-duty vehicle driving on a highway is found using dynamic programming(DP). The solution to an optimal control problem can either be precomputed and used as a trajectory to be tracked by a controller, or it can be found online while driving. Finding a solution using DP is often computationally heavy due to the curse of dimensionality, i.e., the fact that the computation time grows exponentially with the number of states and control signals. In order to make it real-time implementable, algorithms

Funding provided by Swedish Governmental Agency for Innovation Systems (VINNOVA) through the FFI program is gratefully acknowledged.

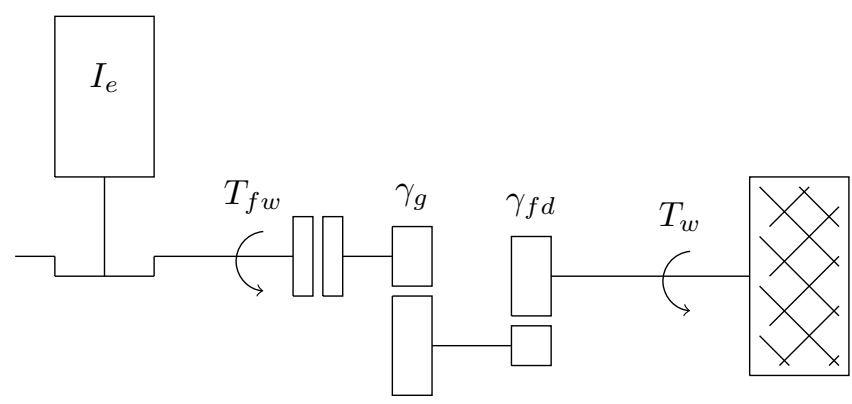

Fig. 1: The powertrain including from the left: engine, flywheel, gearbox, final drive and driving wheel.

reducing the search space using heuristics based on energy and time consumption can be used [4]. In [5], such methods are used for solving a problem regarding the minimization of the fuel consumption in the vicinity of signalized intersections. Another way to reduce the amount of computations performed in the vehicle is to solve the optimal control problem in the cloud [6]. In [7], an optimal cruise control algorithm is developed using DP for a hybrid electrical vehicle having its velocity limited by a driving corridor.

The fact that an engine's efficiency depends on engine speed and torque is considered in the optimization problem in [8]. In [9], an optimization problem involving a vehicle model based on forces is solved by using Pontryagin's maximum principle. The same principle is used in [10], where gear changes are also considered. In [11], the concept of a driving corridor is used, i.e., an upper and a lower limit that the velocity of the vehicle must lie within. The same problem as in [9] is treated, but with more thorough simulations in order to investigate how variations in the driving corridor influence the energy consumption and trip time. The work in this paper extends [9] and [11] by including powertrain components such as an engine and a gearbox in the vehicle model. This is a step towards more implementable solutions, since the tractive force is replaced by the injected fuel and the gear selection is added as a control signal.

The main contribution of this paper is twofold. The first contribution is the application of DP to a driving mission of a heavy-duty truck with varying requirements on the allowed 
TABLE I: Parameters related to the truck and the environment.

\begin{tabular}{|l|c|}
\hline Parameter & Value \\
\hline$m$ - truck mass & $26000 \mathrm{~kg}$ \\
$r_{w}$ - wheel radius & $0.5 \mathrm{~m}$ \\
$P_{\text {max }}$ - maximum power & $250 \mathrm{~kW}$ \\
$c_{d}$ - air drag coefficient & 0.5 \\
$\rho$ - air density & $1.292 \mathrm{~kg} \cdot \mathrm{m}^{-3}$ \\
$A$ - truck cross-sectional area & $10 \mathrm{~m}^{2}$ \\
$c_{r}$ - rolling resistance coefficient & 0.006 \\
$\gamma_{f d}$ - final drive ratio & 2.92 \\
$\omega_{i d l e}$ - idle engine speed & $500 \mathrm{RPM}$ \\
$\omega_{\text {min }}$ - mimimum engine speed & $800 \mathrm{RPM}$ \\
$\omega_{\text {max }}$ - maximum engine speed & $2400 \mathrm{RPM}$ \\
$I_{e}-$ moment of inertia engine & $4 \mathrm{~kg} \cdot \mathrm{m}^{2}$ \\
$I_{w}-$ moment of inertia wheels & $92 \mathrm{~kg} \cdot \mathrm{m}^{2}$ \\
$\tau_{g}$ - time of a gear change & $1 \mathrm{~s}$ \\
$e_{i d e a l}-$ ideal specific energy & $48 \mathrm{MJ} / \mathrm{kg}$ \\
$\gamma_{c}-$ energy to fuel efficiency & 0.45 \\
\hline
\end{tabular}

speed where the boundary conditions are set based on real traffic data. The second contribution is the use of a vehicle model with a fuel map and gearbox losses derived from experiments. This allows the solution to have realistic gear changes and the possibility to coast using neutral gear.

\section{VEHICLE MODEL}

The model of the powertrain of the truck can be seen in Fig. 1 and each part will be described in the following subsections. Parameters related to the truck and the environment are given in Table I.

\section{A. Chassis dynamics}

The dynamics of the truck are given by:

$$
\frac{d v}{d t}=\frac{1}{m c_{m}}\left(\frac{T_{w}}{r_{w}}+F_{e n v}-F_{b}\right)
$$

where $v$ and $m$ are the velocity and mass of the truck, $T_{w}$ is the torque at the wheels, $r_{w}$ is the wheel radius and $F_{b}$ is the force at the brakes. The variable $c_{m}$ represents a mass factor in order to take the moment of inertia of the wheels and engine into account and is given by

$$
c_{m}=\frac{m r_{w}^{2}+I_{w}+\left(\gamma_{g} \gamma_{f d}\right)^{2} I_{e}}{m r_{w}^{2}}
$$

where $I_{w}$ and $I_{e}$ are the moment of inertia of the wheels and engine respectively and $\gamma_{g}$ and $\gamma_{f d}$ are the transmission ratios of the current gear and the final drive respectively. If neutral gear is engaged, then $\gamma_{g}=0$. The sum of the environmental forces $F_{e n v}$ in (1) is given by

$$
F_{e n v}=-m g \cos (\alpha) c_{r}-\frac{1}{2} \rho A c_{d} v^{2}-m g \sin \alpha
$$

where $g$ is the gravitational constant, $\alpha$ is the road slope, $c_{r}$ is the coefficient for the rolling resistance, $\rho$ is the air density, $A$ is the truck frontal area and $c_{d}$ is the air drag coefficient.

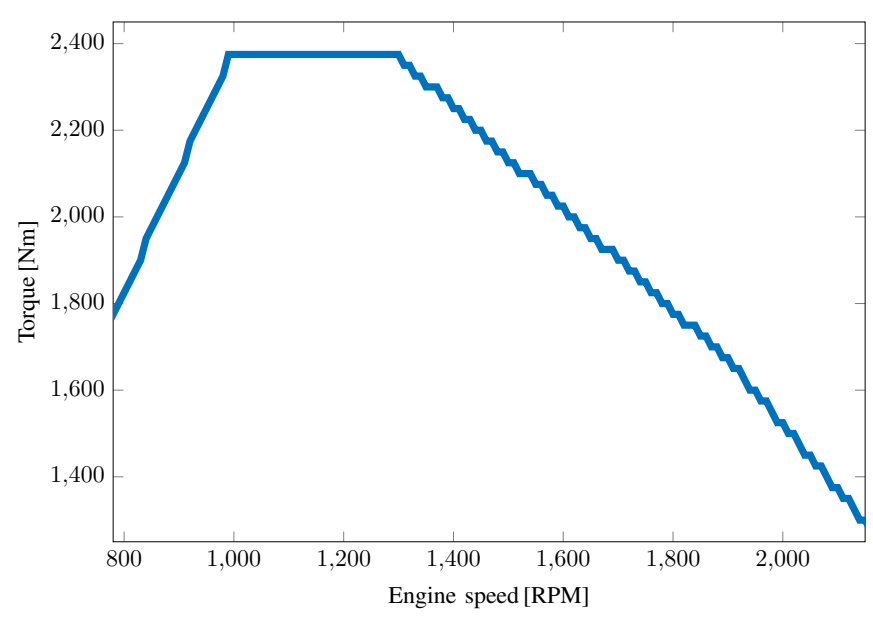

Fig. 2: Maximum flywheel torque $T_{\max }(\omega)$ given as a function of engine speed in revolutions per minute.

\section{B. Engine}

The flywheel torque $T_{f w}$ is given by

$$
T_{f w}=T_{e}-T_{d}
$$

where $T_{e}$ is the engine torque created by the combustion in the engine and $T_{d}$ is the drag torque created by friction in the engine. The flywheel torque $T_{f w}$ is limited such that $T_{f w} \leq$ $T_{\max }(\omega)$ where $T_{\max }(\omega)$ is a function of engine speed $\omega$ that can be seen in Fig. 2. The engine drag torque is a function of engine speed and is taken from previous experiments in a test cell. In the simulations in this paper, it is approximated by the function

$$
T_{d}=d_{0}+d_{1} \omega
$$

where $d_{0}$ and $d_{1}$ are found by a linear least square fit to the experimental values. The minimum possible flywheel torque is negative and is obtained when no fuel is injected to the engine. In this case, the engine torque $T_{e}=0$ and the flywheel torque is created solely by the engine drag torque. The fuel flow $\dot{m}_{f}$ is given in $\mathrm{kg} / \mathrm{s}$ by a fuel map in the form of a look-up table as a function of engine speed and flywheel torque such that

$$
\dot{m}_{f}=\dot{m}_{f}\left(\omega, T_{f w}\right)
$$

This look-up table is taken from previous experiments in test cells. Two specific parts of the fuel map are of special interest. The first is when neutral gear is engaged and is referred to as coasting in neutral. In this case, the engine torque should be enough to overcome the engine drag torque in order to keep the engine speed at the constant value $\omega_{i d l e}$. The fuel consumption is in this case

$$
\dot{m}_{f}=\dot{m}_{f}\left(\omega_{i d l e}, 0\right)
$$

The second case of special interest is when a gear is engaged but no fuel is injected to the engine. This is referred to as coasting with gear and the fuel flow is:

$$
\dot{m}_{f}=0 \text {. }
$$




\section{Gear engaged}

The flywheel torque is transmitted through the gearbox with a resistive torque in the gearbox $T_{g b}$ such that

$$
T_{w}=\left(T_{f w}-T_{g b}\left(\omega, T_{f w}\right)\right) \gamma_{g} \gamma_{f d} .
$$

The torque $T_{g b}$ is given as a function of engine speed and flywheel torque found through experiments. It can be approximated by a linear plane such that

$$
T_{g b}\left(\omega, T_{f w}\right)=k_{1} \omega+k_{2}\left|T_{f w}\right|+k_{3}
$$

for some constants $k_{1}, k_{2}$ and $k_{3}$. The gearbox has 14 gears and four different planes are used to model the losses. The different planes correspond to having high or low range and whether the direct gear is used or not. The engine speed, which is given by the engaged gear and the velocity of the truck, is limited such that

$$
\omega_{\min } \leq \omega \leq \omega_{\max } .
$$

For any velocity of the truck, the set of feasible gears is thus given by the gears satisfying (11).

\section{Neutral gear}

When neutral gear is engaged, the engine speed is constant with the value $\omega_{\text {idle }}$. In order to keep this value, $T_{f w}=$ $T_{e}-T_{d}=0$ must hold. Since only a very small torque is transmitted through the gearbox, the gearbox losses are set to $T_{g b}=0$ when using neutral gear.

\section{E. Gear changes}

A transition from one gear to another is modelled to take $1 \mathrm{~s}$, during which no fuel is injected to the engine. The energy loss due to the engine drag torque is calculated using (5). When a gear shift is performed such that the engine speed is increased, i.e., either from a higher to a lower gear or from neutral gear to any gear, the rotational energy in the engine is increased. The energy required to increase the engine speed to the new value is given by the difference in rotational energy

$$
\Delta E_{\omega}=\frac{I_{e}\left(\omega_{2}^{2}-\omega_{1}^{2}\right)}{2}
$$

for two engine speeds $\omega_{1}$ and $\omega_{2}$. The cost in terms of energy needed in order to increase the engine speed can be related to a fuel cost by a constant $e$ such that $m_{f} e=\Delta E_{\omega}$. The constant $e=e_{\text {ideal }} \gamma_{c}$ is the specific energy of diesel calculated from the ideal specific energy of diesel $e_{\text {ideal }}$ times an average combustion efficiency of the engine $\gamma_{c}$.

\section{PROBLEM FORMULATION}

The state variables, control variables and environmental variables are all discretized with steps of $\Delta s=10 \mathrm{~m}$. The positions $0, \Delta s, 2 \Delta s, \ldots,(N-1) \Delta s$ define the position of the stages. The velocity of the truck is discretized with steps of $\Delta v=0.1 \mathrm{~m} / \mathrm{s}$. The model (1) is discretized using Euler forward such that

$$
x_{k+1}=F_{k}\left(x_{k}, u_{k}\right)
$$

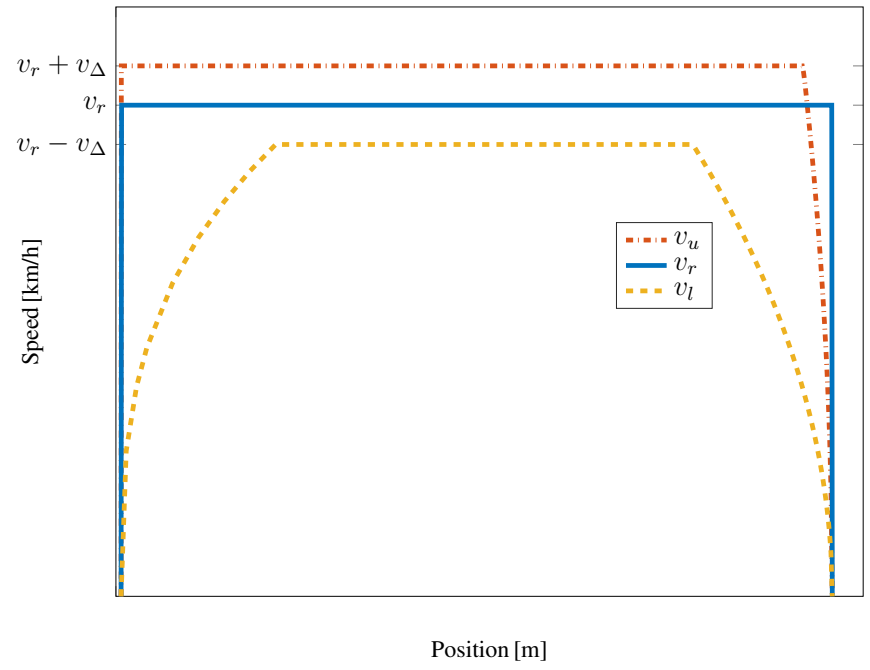

Fig. 3: The driving corridor created from one section of the reference speed trajectory.

where the subscript $k$ indicates the stage and the state variable $x_{k}$ is given by

$$
x_{k}=\left[\begin{array}{ll}
v_{k} & g_{k}
\end{array}\right]^{T}
$$

where $g_{k}$ is the currently engaged gear. The control variable $u_{k}$ is given by

$$
u_{k}=\left[\begin{array}{lll}
T_{e, k} & g_{d, k} & F_{b, k}
\end{array}\right]^{T}
$$

where $g_{d}$ is the control gear. If the control gear is different from the current gear, i.e., $g_{d, k} \neq g_{k}$, then a gear change is requested. The velocity is at all positions bounded by the driving corridor such that $v_{l, k} \leq v_{k} \leq v_{u, k}$, where $v_{l}$ and $v_{u}$ are the lower and upper bound of the driving corridor. As discussed in [11], the driving corridor is created from a reference speed trajectory $v_{r}$ and from real truck operation data. The main idea of using traffic data is to restrict the truck to decelerate in a way that does not deviate too much from a normal way of driving. Two inputs are needed in order to create the driving corridor: the maximum and minimum allowed deviation from the reference speed trajectory $v_{\Delta}$ and the number of standard deviations $n_{\Sigma}$ the truck is allowed to deviate from the mean deceleration when $v_{r}$ is decreased. The creation of the driving corridor for one section of the driving cycle can be seen in Fig. 3. The lower limit $v_{l}$ is not set based on traffic data during the acceleration phase. Instead, it is set using $60 \%$ of maximum power, which was found empirically to be a good value during simulations.

The objective of the optimization problem in this paper is the minimization of the weighted sum

$$
\sum_{k=1}^{N-1} m_{f, k}+\beta t_{k}
$$

where $\beta$ defines the weighting between fuel and the time $t$. 


\section{DYNAMIC PROGRAMMING SOLUTION}

\section{A. Theoretical background}

The problem formulated in this paper is solved by using DP introduced in [12]. The main idea is to use the principle of optimality, i.e., the fact that regardless of what the initial state is, the remaining control must be optimal with respect to the states resulting from this control. The optimal cost-to-go $J_{k}\left(x_{k}\right)$ is defined as the cost for taking the truck from the state $x_{k}$ at the current stage to the final stage. The transition cost from one stage to the next is denoted by $\zeta_{k}\left(x_{k}, u_{k}\right)$. The algorithm becomes:

1) At the last stage $N$ of the driving mission, the velocity $v_{N}$ is fixed while the gear $g_{N}$ is only restricted by the requirements for the engine speed (11). The optimal costto-go is equal to zero for all feasible states at this stage.

2) For $k=N-1, \ldots, 1$, the optimal cost-to-go is given by

$$
J_{k}\left(x_{k}\right)=\min _{u_{k}}\left\{\zeta_{k}\left(x_{k}, u_{k}\right)+J_{k+1}\left(F_{k}\left(x_{k}, u_{k}\right)\right)\right\} .
$$

3) The solution constitutes of the control sequence $u_{k}$ for $k=1, \ldots, N-1$.

The DP algorithm in this paper considers four different types of transitions between stages described in the following subsections.

\section{B. Same gear transitions}

For each velocity at the start stage, the set of feasible gears is given by the constraints on the engine speed. Then, for each velocity for which a gear is feasible also at the target state, the required flywheel torque $T_{f w}$ and/or braking force $F_{b}$ required for this transition is calculated using (13). The optimal cost-to-go and the control signals are given by

$$
J_{k}(x)=\min _{u_{k}}\left\{\zeta_{k}\left(x_{k}, u_{k}\right)+J_{k+1}\left(F_{k}\left(x_{k}, u_{k}\right)\right)\right\}
$$

where $\zeta_{k}\left(x_{k}, u_{k}\right)$ is given by

$$
\zeta_{k}(x, u)=\left(\dot{m}_{f, k}+\beta\right) t_{k} .
$$

The fuel mass flow $\dot{m}_{f, k}$ is given by (6) and the evolved time is given by

$$
t_{k}=\frac{2 \Delta s}{v_{k}+v_{k+1}} .
$$

\section{Coasting in neutral}

A transition to a state where neutral gear is engaged is referred to as coasting in neutral. This is performed during a full interval $\Delta s$. The resulting velocity at the next stage is in most cases not equal to any of the beforehand discretized velocities. The cost-to-go at the end of one step of coasting in neutral is therefore given by a linear interpolation of the two surrounding values of the discretized velocities. The control signals are $g_{d, k}=0$ and $T_{e, k}=T_{d}\left(\omega_{i d l e}\right)$ and the braking force $F_{b, k}$ is only applied in order to avoid overspeeding. The optimal cost-to-go at stage $k$ is given by

$$
\begin{aligned}
& J_{k}(x)=\min _{u_{k} \in U_{k}}\left\{\zeta_{k}\left(x_{k}, u_{k}\right)\right. \\
& \left.+\epsilon J_{k+1}\left(\overline{F_{k}\left(x_{k}, u_{k}\right)}\right)+(1-\epsilon) J_{k+1}\left(\underline{F_{k}\left(x_{k}, u_{k}\right)}\right)\right\}
\end{aligned}
$$

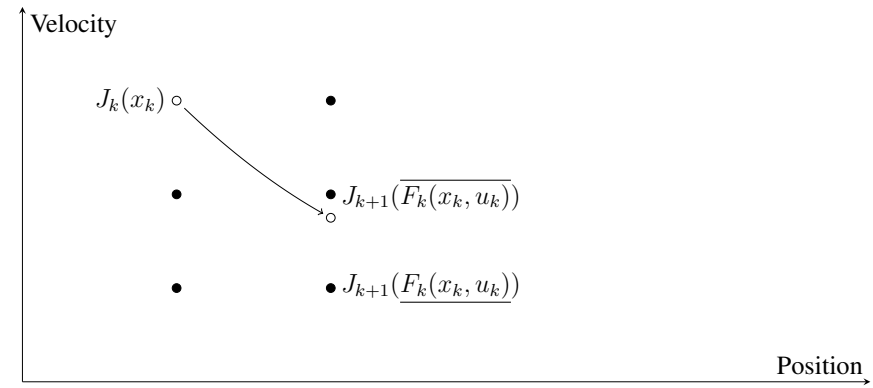

(a) Transition using coasting in neutral or coasting with gear.

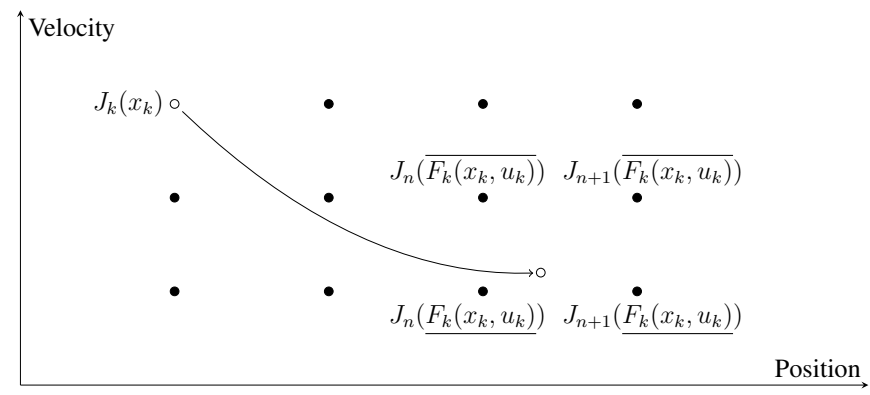

(b) Transition involving a gear change.

Fig. 4: Transitions from stage $k$ for which interpolation of the cost-to-go and control signals are necessary.

where $\overline{F_{k}\left(x_{k}, u_{k}\right)}$ and $F_{k}\left(x_{k}, u_{k}\right)$ are the state $F_{k}\left(x_{k}, u_{k}\right)$ with its velocity rounded to the nearest higher and lower value respectively and $\epsilon$ is given by the linear interpolation between the two states, see Fig. 4a. The transition cost $\zeta_{k}\left(x_{k}, u_{k}\right)$ is given by (19) with the fuel mass flow given by (7).

\section{Coasting with gear}

Transitions using coasting with gear are also performed during a full interval $\Delta s$, but with lower end velocity than when coasting in neutral, since $T_{f w}<0$ in this case. The optimal cost-to-go is calculated for each feasible velocity and gear using (21) and (19) with the fuel mass flow given by (8). The control signals are $g_{d, k}=g_{k}, T_{e, k}=0$ and the braking force $F_{b, k}$ is only applied in order to avoid overspeeding.

\section{E. Gear changes}

A gear shift always starts at a position belonging to a stage, lasts for $\tau_{g}$, and might therefore end between two stages. During a gear shift, the truck rolls with open clutch, i.e., with zero flywheel torque. If the truck reaches the next stage in less than $\tau_{g}$, the residual time of the gear change is calculated until two stages are found between which the gear change is completed. The cost-to-go is then given by a bilinear interpolation between the four surrounding points in terms of position and velocity, which can be seen in Fig. $4 \mathrm{~b}$. The optimal cost-to-go from a specific state $x_{k}$ ending between stage $n$ and $n+1$ is given by

$$
\begin{aligned}
& J_{k}(x)=\min _{u_{k}}\left\{\zeta_{k}\left(x_{k}, u_{k}\right)\right. \\
& +\epsilon_{1} J_{n}\left(\overline{F_{k}\left(x_{k}, u_{k}\right)}\right)+\epsilon_{2} J_{n}\left(F_{k}\left(x_{k}, u_{k}\right)\right) \\
& \left.+\epsilon_{3} J_{n+1}\left(\overline{F_{k}\left(x_{k}, u_{k}\right)}\right)+\epsilon_{4} J_{n+1}\left(F_{k}\left(x_{k}, u_{k}\right)\right)\right\}
\end{aligned}
$$



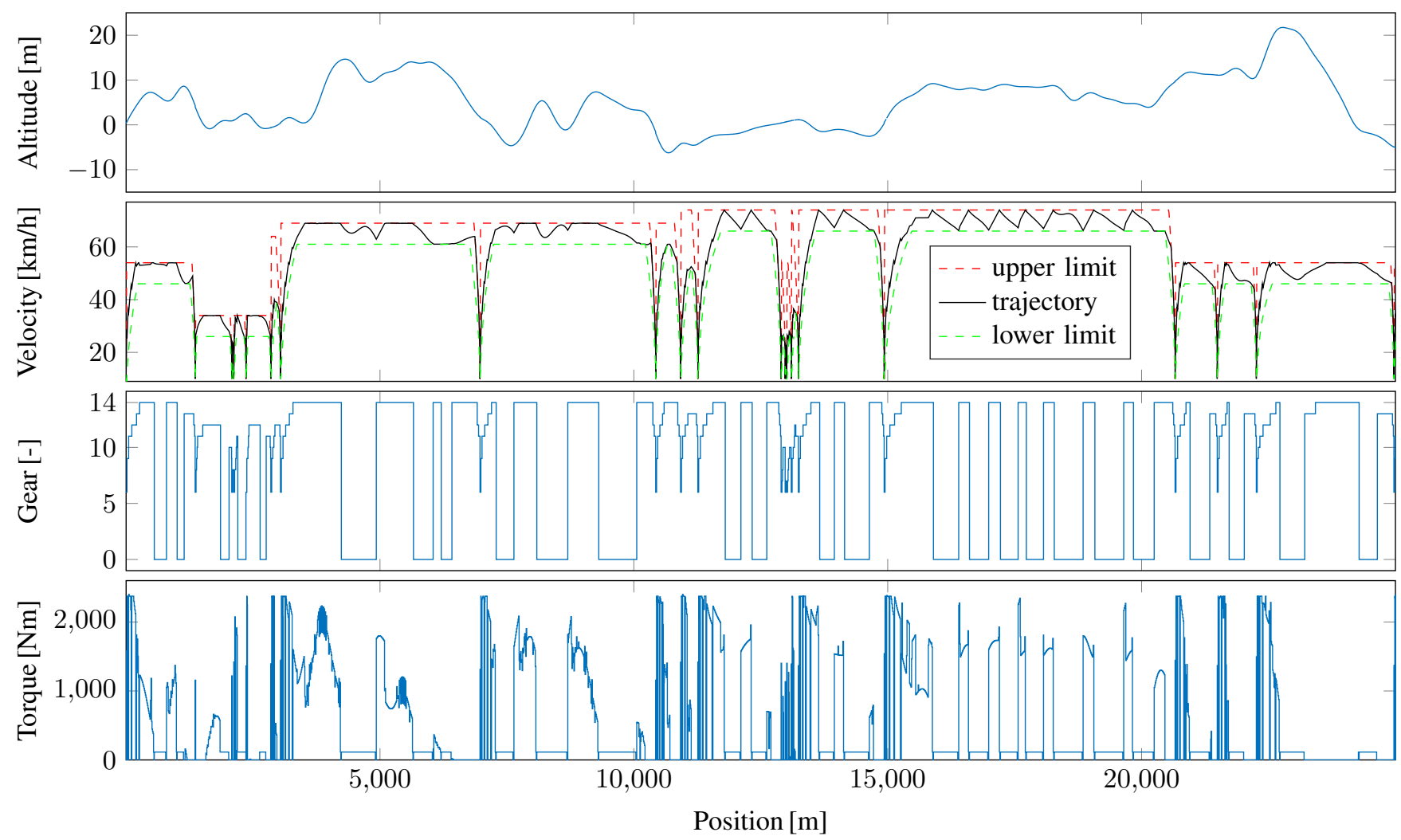

(a) Simulation using $\Delta_{v}=4 \mathrm{~km} / \mathrm{h}$ and $n_{\Sigma}=1 \mathrm{std}$.
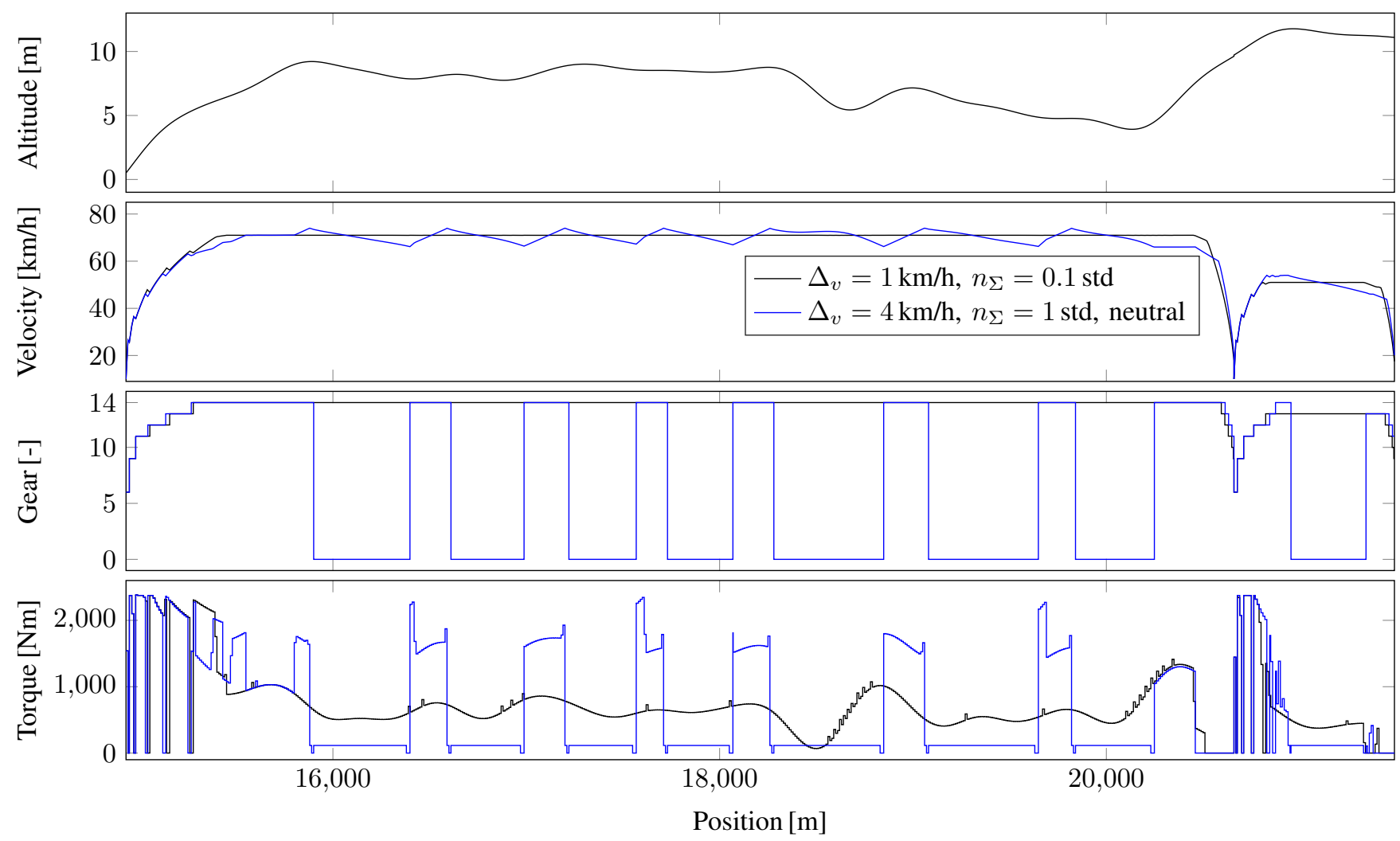

(b) Trajectory using a narrow corridor compared to using a wider corridor and the ability to coast in neutral gear.

Fig. 5: Simulation results showing altitude, velocity trajectories, gear and engine torque. 
where $\epsilon_{i}$ are constants defined by the bilinear interpolation and $\sum_{i=1}^{4} \epsilon_{i}=1$. The control gear is any gear not equal to either the current gear nor neutral gear, $T_{e, k}=0$ and the braking force $F_{b, k}$ is only applied in order to avoid overspeeding. The transition cost to the position where gear change is finished becomes

$$
\zeta_{k}\left(x_{k}, u_{k}\right)=\sum_{i=k}^{n}\left(\dot{m}_{f, i}+\beta\right) t_{i}+\gamma \Delta E_{\omega}
$$

where $t_{i}$ is given by (20) for $i=k, \ldots, n-1$ and $t_{n}$ is the time consumed at the incomplete last stage such that $\sum_{i=k}^{n} t_{i}=\tau_{g}$. The fuel mass flow is zero for an upshift, since the decrease in engine speed is used for overcoming the engine drag torque. When changing from a higher gear or from neutral gear, the fuel mass flow in (23) is set by $\dot{m}_{f}\left(\omega_{k}, 0\right)$.

\section{F. Solution}

The steps in IV-A are applied to IV-B - IV-E. If no feasible solution from a state is found, the cost-to-go is set to infinity. The control vector $u_{k}$ is stored for each feasible state at each stage. When the backward DP-algorithm is finished, a forward algorithm using the optimal control $u_{k}$ for the current state is applied. Using the control $u_{k}$, the truck might end up in between two discretized velocities during gear changes, coasting in neutral or coasting with gear. If the current state is between discretized states with the same gear, interpolation is used in order to find the control at this state. If the current state is between discretized states with different gears, the control belonging to the nearest one in terms of velocity is chosen.

\section{Simulation Result}

Simulations were performed using Matlab on a $66 \mathrm{~km}$ driving cycle developed for testing distribution driving. The driving cycle includes an altitude profile and a reference speed trajectory. The cycle contains a total of 53 stops, at which the truck is starting and stopping with a velocity of $10 \mathrm{~km} / \mathrm{h}$, since the dynamics during very small velocities are out of scope for this paper. The intention of the simulations is to investigate how the fuel consumption is affected by a wider driving corridor and by the possibility to use neutral gear. In order to fairly compare simulations with different driving corridors, the value of the time penalty parameter $\beta$ is adjusted such that the simulations have the same trip time. A simulation with a narrow driving corridor $\Delta_{v}=1 \mathrm{~km} / \mathrm{h}$ and $n_{\Sigma}=0.1$ is used as a benchmark. The resulting trajectory using a wider driving corridor with the possibility to use neutral gear can be seen in Fig. 5a for the first $25 \mathrm{~km}$ of the cycle. A trajectory from the same simulation is in Fig. 5b shown together with a trajectory from the benchmark solution with the same trip time. The resulting fuel consumption for trajectories with the same trip time, is summarized in Table II.

\section{DISCUSSION}

By using the truck model and problem formulation in this paper, the optimal control in terms of gear selection and engine torque can be found using DP. The simulations show potential
TABLE II: Resulting fuel consumption for five different simulations with the same trip time.

\begin{tabular}{l|l|l} 
Driving corridor & Neutral allowed & Fuel savings \\
\hline$\Delta_{v}=1 \mathrm{~km} / \mathrm{h}, n_{\Sigma}=0.1 \mathrm{std}$ & no & $0 \%$ \\
$\Delta_{v}=2 \mathrm{~km} / \mathrm{h}, n_{\Sigma}=1 \mathrm{std}$ & no & $5.0 \%$ \\
$\Delta_{v}=4 \mathrm{~km} / \mathrm{h}, n_{\Sigma}=1 \mathrm{std}$ & no & $8.0 \%$ \\
$\Delta_{v}=2 \mathrm{~km} / \mathrm{h}, n_{\Sigma}=1 \mathrm{std}$ & yes & $9.2 \%$ \\
$\Delta_{v}=4 \mathrm{~km} / \mathrm{h}, n_{\Sigma}=1 \mathrm{std}$ & yes & $12.7 \%$
\end{tabular}

fuel savings of up to $12.7 \%$ without increasing the trip time. It can be seen in Fig. 5a that if allowed, coasting in neutral will be used frequently. Even on flat regions, the truck will switch between high torque and coasting in neutral, which is known as Pulse-and-Gliding [8]. Since the truck may only deviate within the driving corridor, the frequency at which neutral gear is engaged depends on the width of the driving corridor. The computation time of the proposed algorithm has not been considered in this paper but will be investigated in future work. This can be done by either taking means to make the algorithm real-time implementable or to use the derived solutions as trajectories for an online controller to track.

\section{REFERENCES}

[1] "Scania Active Prediction," Scania CV AB, Tech. Rep. December, 2012. [Online]. Available: https://www.scania.com/group/en/event/pressroomscania-active-prediction/

[2] A. Sciarretta, G. De Nunzio, and L. L. Ojeda, "Optimal Ecodriving Control: Energy-Efficient Driving of Road Vehicles as an Optimal Control Problem," IEEE Control Systems Magazine, vol. 35, no. 5, pp. 71-90, 2015.

[3] E. Hellström, "Look-ahead Control of Heavy Vehicles," Ph.D. dissertation, Linköping University, 2010.

[4] O. Chevrant-Breton, T. Guan, and C. W. Frey, "Search space reduction in dynamic programming using monotonic heuristics in the context of model predictive optimization," in 2014 IEEE 17th International Conference on Intelligent Transportation Systems, Qingdao, 2014, pp. 2113-2118.

[5] R. K. Kamalanathsharma and H. A. Rakha, "Multi-stage dynamic programming algorithm for eco-speed control at traffic signalized intersections," in 2013 IEEE 16th International Conference on Intelligent Transportation Systems, The Hague, 2013, pp. 2094-2099.

[6] E. Ozatay, S. Onori, J. Wollaeger, U. Ozguner, G. Rizzoni, D. Filev, J. Michelini, and S. D. Cairano, "Cloud-Based Velocity Profile Optimization for Everyday Driving : A Dynamic-Programming-Based Solution," IEEE Transactions on Intelligent Transportation Systems, vol. 15, no. 6, pp. 2491-2505, 2014.

[7] H.-G. Wahl, K.-L. Bauer, F. Gauterin, and M. Holzapfel, "A realtime capable enhanced dynamic programming approach for predictive optimal cruise control in hybrid electric vehicles," in 2013 IEEE 16th International Conference on Intelligent Transportation Systems, The Hague, 2013, pp. 1662-1667.

[8] S. E. Li and H. Peng, "Strategies to Minimize Fuel Consumption of Passenger Cars during Car-Following Scenarios," in 2011 American Control Conference, San Francisco, 2011, pp. 2107-2112.

[9] M. Henriksson, O. Flärdh, and J. Mårtensson, "Optimal Speed Trajectory for a Heavy Duty Truck Under Varying Requirements," in 2016 IEEE 19th International Conference on Intelligent Transportation Systems, Rio de Janeiro, 2016, pp. 820-825.

[10] B. Passenberg, P. Kock, and O. Stursberg, "Combined time and fuel optimal driving of trucks based on a hybrid model," in European control conference 2009, Budapest, 2009.

[11] M. Henriksson, O. Flärdh, and J. Mårtensson, "Optimal Speed Trajectories Under Variations in the Driving Corridor," in 20:th IFAC World Congress, Toulouse, 2017, pp. 13 062-13067.

[12] R. Bellman, Dynamic Programming, ser. Dover Books on Computer Science Series. Princeton, N.J. : Princeton Univ. Press, 1957. 